\title{
Fractal Logistic Equation
}

\author{
Alireza Khalili Golmankhaneh ${ }^{1, *(D)}$ and Carlo Cattani ${ }^{2}$ (D) \\ 1 Department of Physics, Urmia Branch, Islamic Azad University, Urmia, PO Box 969, Iran \\ 2 Engineering School, DEIM, Tuscia University, Viterbo, Italy and Ton Duc Tang University, HCMC, \\ Ho Chí Minh 758307, Vietnam \\ * Correspondence: alirezakhalili2002@yahoo.co.in
}

Received: 4 June 2019; Accepted: 8 July 2019; Published: 11 July 2019

\begin{abstract}
In this paper, we give difference equations on fractal sets and their corresponding fractal differential equations. An analogue of the classical Euler method in fractal calculus is defined. This fractal Euler method presets a numerical method for solving fractal differential equations and finding approximate analytical solutions. Fractal differential equations are solved by using the fractal Euler method. Furthermore, fractal logistic equations and functions are given, which are useful in modeling growth of elements in sciences including biology and economics.
\end{abstract}

Keywords: fractal calculus; fractal difference equations; fractal logistic function; fractal logistic equation

\section{Introduction}

Fractal geometry includes shapes which are scale invariant and have fractional dimensions and self-similar properties [1-17]. Analysis on fractals was formulated using different methods such as harmonic analysis, probabilistic methods, measure theory, fractional calculus, fractional spaces, and time-scale calculus [18-29].

Fractional calculus is non-local which provides analogues of local models in science and engineering that found many applications [30-35].

The logistic equation and modified logistic equation have been applied to model growth of population, durable consumer goods, forecasting many social and technological patterns. The growth curves to the biological, technological and economic fields fitted by the logistic function. The differential form of the logistic growth equation was originally developed by Verhulst in 1838 [36]. A modified logistic model was proposed by Blumberg [37]; unlike the original logistic model, this cannot be integrated analytically but only solved numerically. Data analysis fits the modified logistic model [38-40].

Fractional difference and logistic equations were studied by many researchers [41-56].

Recently, in the seminal papers [57,58], generalized standard calculus was adopted to include functions with support on totally disconnected fractal sets and self-avoiding curves. Fractal calculus was applied as a mathematical model for diffraction of light and for random walks which give anomalous diffusion on fractal Cantor-like sets [59-61]. Fractal Sumudu transforms were defined, which are important in control engineering problems [62,63].

In this work, we present that which corresponds to a given fractal difference equation fractal differential equation. We provide analogues of the numerical method for finding the solutions of the fractal differential equations such as the fractal logistic equation.

The framework of the paper is as follows:

In Section 2 we review some basic tools. We define the fractal shift operator and fractal difference operator in Section 3. In Section 4 we use these operators to define fractal difference equations and the corresponding fractal differential equations. The fractal Euler method, which is used as a numerical method for solving fractal differential equations, is given in Section 5. In Section 6 we present the fractal logistic equation and function and conclusions are given in Section 7. 


\section{Basic Tools}

In this section, we summarize the local generalized Riemman calculus on the fractal middle- $\kappa$ Cantor set.

\subsection{Middle- $\kappa$ Cantor Set}

The middle- $\kappa$ Cantor set is generated by the following steps:

1-Step 1. Pick up an open interval of length $0<\kappa<1$ from the middle of the $I=[0,1]$.

$$
C_{1}^{\kappa}=\left[0, \frac{1}{2}(1-\kappa)\right] \cup\left[\frac{1}{2}(1+\kappa), 1\right] .
$$

2-Step 2. Delete disjoint open intervals of length $\kappa$ from the middle of the remaining closed intervals of step 1.

$$
\begin{aligned}
& C_{2}^{\kappa}=\left[0, \frac{1}{4}(1-\kappa)^{2}\right] \cup\left[\frac{1}{4}\left(1-\kappa^{2}\right), \frac{1}{2}(1-\kappa)\right] \cup\left[\frac{1}{2}(1+\kappa)+\frac{1}{2}((1+\kappa)\right. \\
& \left.\left.+\frac{1}{2}(1-\kappa)^{2}\right)\right] \cup\left[\frac{1}{2}(1+\kappa)\left(1+\frac{1}{2}(1-\kappa)\right), 1\right]
\end{aligned}
$$

3-Step $m$. Remove disjoint open intervals of length $\kappa$ from the middle of the remaining closed intervals of step $m-1$.

$$
C^{\kappa}=\bigcap_{m=1}^{\infty} C_{m}^{\kappa}
$$

Note that the measure of middle- $\kappa$ Cantor sets is zero [64].

For every middle- $\kappa$ Cantor set which generally is called Cantor-like sets, the Hausdorff dimension is given by

$$
\operatorname{dim}_{H}\left(C^{\kappa}\right)=\frac{\log 2}{\log 2-\log (1-\kappa)}
$$

where $H\left(C^{\kappa}\right)$ is the Hausdorff measure which was used to derive Hausdorff dimension [64].

\subsection{Local Fractal Calculus}

If $C^{\kappa}$ is middle- $\kappa$ Cantor set then the flag function is defined by $[57,58,60]$,

$$
F\left(C^{\kappa}, J\right)= \begin{cases}1, & \text { if } C^{\kappa} \cap J \neq \varnothing ; \\ 0, & \text { otherwise, }\end{cases}
$$

where $J=\left[b_{1}, b_{2}\right]$. Then, $\mathcal{P}^{\alpha}\left[C^{\mathcal{K}}, W\right]$ is given in $[57,58,60]$ by

$$
\mathcal{P}^{\alpha}\left[C^{\kappa}, W\right]=\sum_{i=1}^{n} \Gamma(\alpha+1)\left(t_{i}-t_{i-1}\right)^{\alpha} F\left(C^{\kappa},\left[t_{i-1}, t_{i}\right]\right),
$$

where $W_{\left[b_{1}, b_{2}\right]}=\left\{b_{1}=t_{0}, t_{1}, t_{2}, \ldots, t_{n}=b_{2}\right\}$ is a subdivisions of $J$.

The mass function $\gamma^{\alpha}\left(C^{\kappa}, b_{1}, b_{2}\right)$ is defined in $[57,58,60]$ by

$$
\gamma^{\alpha}\left(C^{\kappa}, b_{1}, b_{2}\right)=\lim _{\delta \rightarrow 0} \gamma_{\delta}^{\alpha}=\lim _{\delta \rightarrow 0}\left(\inf _{E_{[r, t]}:|E| \leq \delta} \mathcal{P}^{\alpha}\left[C^{\mathcal{K}}, E\right]\right),
$$

here, infimum is taking over all subdivisions $E$ of $\left[b_{1}, b_{2}\right]$ satisfying $|E|:=\max _{1 \leq i \leq n}\left(t_{i}-t_{i-1}\right) \leq \delta$. 
The integral staircase function $S_{C^{k}}^{\alpha}(t)$ is defined in $[57,58]$ by

$$
S_{C^{\kappa}}^{\alpha}(t)= \begin{cases}\gamma^{\alpha}\left(C^{\kappa}, b_{0}, t\right), & \text { if } t \geq b_{0} \\ -\gamma^{\alpha}\left(C^{\kappa}, b_{0}, t\right), & \text { otherwise }\end{cases}
$$

where $b_{0}$ is an arbitrary and fixed real number.

The $\gamma$-dimension of a set $C^{\kappa} \cap\left[b_{1}, b_{2}\right]$ is defined

$$
\begin{aligned}
\operatorname{dim}_{\gamma}\left(C^{\mathcal{K}} \cap\left[b_{1}, b_{2}\right]\right) & =\inf \left\{\alpha: \gamma^{\alpha}\left(C^{\kappa}, b_{1}, b_{2}\right)=0\right\} \\
& =\sup \left\{\alpha: \gamma^{\alpha}\left(C^{\kappa}, b_{1}, b_{2}\right)=\infty\right\} .
\end{aligned}
$$

The $C^{\alpha}$-limit of a function $g: \Re \rightarrow \Re$ is given by

$$
\forall \epsilon>0, \exists \delta>0 \quad z \in C^{\kappa} \text { and }|z-t|<\delta \Rightarrow|g(z)-\ell|<\epsilon \text {. }
$$

If $\ell$ exists, then we have

$$
\ell=C^{\alpha}-\lim _{z \rightarrow t} g(z)
$$

The $C^{\alpha}$-continuity of a function $k: \Re \rightarrow \Re$ is defined by

$$
g(t)=C^{\alpha}-\lim _{z \rightarrow t} g(z)
$$

The $C^{\alpha}$-derivative of $f(t)$ at $t$ is defined [57]

$$
D_{C^{\kappa}}^{\alpha} f(t)= \begin{cases}C^{\alpha}-\lim _{y \rightarrow t} \frac{f(y)-f(t)}{S_{C^{k}}^{\alpha}(y)-S_{C^{k}}^{\alpha}(t)}, & \text { if, } t \in C^{\kappa} \\ 0, & \text { otherwise. }\end{cases}
$$

if the limit exists.

The $C^{\alpha}$-integral of $k(t)$ on $J=\left[b_{1}, b_{2}\right]$ is defined in $[57,58,60]$ and approximately given by

$$
\int_{b_{1}}^{b_{2}} f(t) d^{\alpha} t \approx \sum_{i=1}^{n} f\left(t_{i}\right)\left(S_{C^{\kappa}}^{\alpha}\left(t_{i}\right)-S_{C^{k}}^{\alpha}\left(t_{i-1}\right)\right) .
$$

For more details we refer the reader to $[57,58]$.

The Characteristic function of the middle- $\kappa$ Cantor set is defined in [60] by

$$
\chi_{C^{k}}(\alpha, t)= \begin{cases}\frac{1}{\Gamma(\alpha+1)}, & t \in C^{\kappa} ; \\ 0, & \text { otherwise. }\end{cases}
$$

Some important formulas:

$$
\begin{aligned}
D_{C^{\kappa}}^{\alpha} \chi_{C^{\kappa}}(\alpha, t) t & =\frac{1}{\Gamma(\alpha+1)} \chi_{C^{\kappa}}(\alpha, t), \\
D_{C^{\kappa}}^{\alpha} \chi_{C^{\kappa}}(\alpha, t) t^{2} & =\frac{2}{\Gamma(\alpha+1)} \chi_{C^{\kappa}}(\alpha, t) t, \\
D_{C^{\kappa}}^{\alpha} \sin \left(t \chi_{C^{\kappa}}(\alpha, t)\right) & =\frac{1}{\Gamma(\alpha+1)} \cos \left(t \chi_{C^{\kappa}}(\alpha, t)\right) .
\end{aligned}
$$

In Figure 1 we have clarified the definitions of Section 2. 


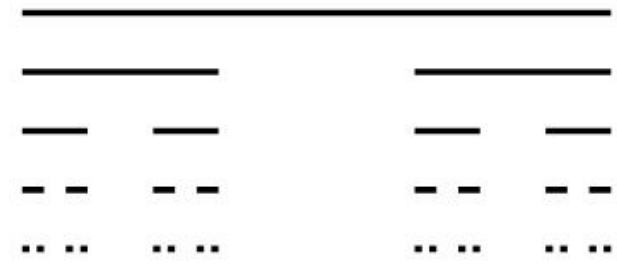

(a) Middle- $\kappa$ Cantor set with $\kappa=1 / 3$

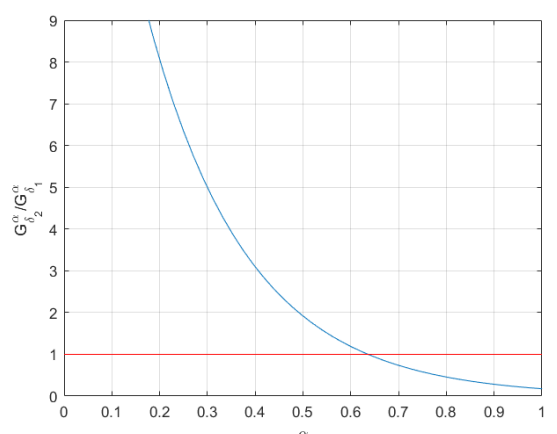

(c) The $\gamma$-dimension gives $\alpha=0.5$ to middle- $\kappa$ Cantor set with $\kappa=1 / 3$

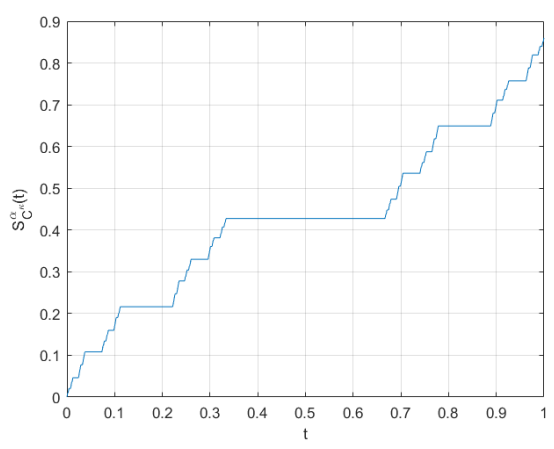

(b) Staircase function corresponding to middle- $\kappa$ Cantor set with $\kappa=1 / 3$

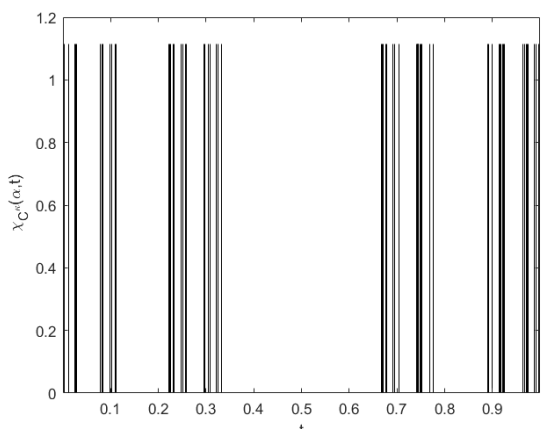

(d) Characteristic function for middle- $\kappa$ Cantor set with $\kappa=1 / 3$

Figure 1. Figures for the Section 2.

\section{Fractal Finite Difference and Fractal Derivative}

In this section, we define fractal shift operator and fractal difference operator. Furthermore, the fractal differential equation and its corresponding difference equation is given. Let us define a $\alpha$-difference equation on the fractal middle- $\eta$ Cantor set as follows:

$$
z_{K, n+1}=\chi_{K} B z_{K, n},
$$

where $C^{\kappa}=K$ and $B$ is an operator. In view of fractal Taylor expansion, we have

$$
\begin{aligned}
z_{K, n+1} & =\left.\sum_{k=0}^{\infty} \frac{S_{K}^{\alpha}(h)^{k}}{k !}\left(D_{K}^{\alpha}\right)^{k} z(t)\right|_{t=t_{n}} \\
& =e^{S_{K}^{\alpha}(h) D_{K}^{\alpha} z_{n},}
\end{aligned}
$$

where

$$
\begin{aligned}
z_{n+1} & =z\left(t_{n+1}\right), \\
t_{n+1} & =h+t_{n}, \quad h \in \Re, \quad z_{n} \in K, \\
D_{K}^{\alpha} z_{K, n} & =\left.D_{K}^{\alpha} z_{K}(t)\right|_{t=t_{n}},
\end{aligned}
$$

consequently, we have

$$
B=e^{S_{K}^{\alpha}(h) D_{K}^{\alpha}} .
$$

In view of Equation (16), $B$ is called fractal shift operator. 
Fractal difference operator is denoted by $\Delta_{K}$ and defined by

$$
\Delta_{K}=B-1,
$$

it follows that

$$
\Delta_{K} z_{n}=z_{n+1}-z_{n} .
$$

Using Equation (18) the fractal $\alpha$-order derivative in terms of the fractal $\alpha$-difference equation is

$$
\begin{aligned}
D_{K}^{\alpha} & =\frac{1}{S_{K}^{\alpha}\left(h_{n}\right)} \ln B \\
& =\frac{1}{S_{K}^{\alpha}(h)} \ln \left(1+\Delta_{K}\right) \\
& =\frac{1}{S_{K}^{\alpha}(h)} \sum_{k=1}^{\infty}(-1)^{k+1} \frac{\Delta_{K}^{k}}{k !} .
\end{aligned}
$$

Fractal higher $\alpha$-order derivatives is approximated by

$$
\left(D_{K}^{\alpha}\right)^{m} \approx\left(\frac{\Delta_{K}}{S_{K}^{\alpha}(h)}\right)^{m} .
$$

Example 1. Consider $z(t)=S_{K}^{\alpha}(t)$, then using Equation (16) we can write

$$
\begin{aligned}
S_{K, n+1}^{\alpha} & =\chi_{K} B S_{K, n}^{\alpha} \\
& =\left.\sum_{k=0}^{\infty} \frac{S_{K}^{\alpha}(h)^{k}}{k !}\left(D_{K}^{\alpha}\right)^{k} S_{K}^{\alpha}(t)\right|_{t=t_{n}} \\
& =S_{K}^{\alpha}\left(t_{n}\right)+S_{K}^{\alpha}(h) \chi_{K}\left(t_{n}\right),
\end{aligned}
$$

where

$$
D_{K}^{\alpha} S_{K, n}^{\alpha}=\left.D_{K}^{\alpha} S_{K}^{\alpha}(t)\right|_{t=t_{n}}=\chi_{K}\left(t_{n}\right)
$$

By Equation (20) we have

$$
\Delta_{K} S_{K, n}^{\alpha}=S_{K, n+1}^{\alpha}-S_{K, n}^{\alpha} .
$$

Using Equation (21) we get

$$
\begin{aligned}
\left(D_{K}^{\alpha}\right)^{2} S_{K}^{\alpha}(t) & =\frac{1}{S_{K}^{\alpha}(h)^{2}}\left(\Delta_{K}\right)^{2} S_{K, n}^{\alpha} \\
& =\frac{1}{S_{K}^{\alpha}(h)^{2}} \Delta_{K}\left(S_{K, n+1}^{\alpha}-S_{K, n}^{\alpha}\right) \\
& =\frac{1}{S_{K}^{\alpha}(h)^{2}}\left(S_{K, n+2}^{\alpha}-2 S_{K, n+1}^{\alpha}+S_{K, n}^{\alpha}\right) .
\end{aligned}
$$

We note that Equation (24) is used to obtain numerical solution of the second $\alpha$-order fractal differential equations.

\section{Fractal Difference and Differential Equations}

In this section, we show the relation between fractal difference equations and fractal differential equations which should later be used to find numerical solution of the fractal differential equations.

Consider the following fractal $\alpha$-difference equation

$$
z_{n+1}=\chi_{K} \mathbf{B} z_{n}, \quad z_{n} \in K, \quad \mathbf{B} \in \Re,
$$


then the solution of Equation (25) is

$$
z_{n}=\chi_{K} \mathbf{B}^{n} z_{0}
$$

If we set $z_{n}=z\left(t_{n}\right)$, then

$$
S_{K}^{\alpha}\left(t_{n+1}\right)=S_{K}^{\alpha}\left(t_{n}\right)+S_{K}^{\alpha}(h), \quad t_{n}, \quad t_{n+1} \in K, \quad h \in(0,1),
$$

and

$$
S_{K}^{\alpha}\left(t_{n}\right)=n S_{K}^{\alpha}(h)
$$

It follows that Equation (25) gives

$$
\frac{z\left(t_{n}+h\right)-z\left(t_{n}\right)}{S_{K}^{\alpha}(h)}=\frac{\mathbf{B}-1}{S_{K}^{\alpha}(h)} z\left(t_{n}\right),
$$

then the corresponding fractal differential equation is

$$
D_{K}^{\alpha} z(t)=\frac{\mathbf{B}-1}{S_{K}^{\alpha}(h)} z(t) .
$$

The solution of Equation (30) is

$$
z(t)=z(0) \exp \left(\frac{\mathbf{B}-1}{S_{K}^{\alpha}(h) S_{K}^{\alpha}(t)}\right) .
$$

\section{Numerical Method for Solving Fractal Differential Equation}

In this section, we present an analogue of Euler method in the fractal calculus by using fractal difference operator.

Let us consider $\alpha$-order fractal differential equation as

$$
D_{K}^{\alpha} z(t)=f\left(S_{K}^{\alpha}(t), z(t)\right), \quad z\left(t_{0}\right)=z_{0} .
$$

In view of Equation (21) we obtain

$$
z_{n+1}(t)=z_{n}(t)+S_{K}^{\alpha}(h) f\left(S_{K}^{\alpha}(t), z(t)\right) .
$$

By substituting fractal Taylor expansion of $f\left(S_{K}^{\alpha}(t), z(t)\right)$, in Equation (33) we get approximate analytical solution of Equation (32) as follows:

$$
z(t+h)=z\left(t_{0}\right)+S_{K}^{\alpha}(h) D_{K}^{\alpha} z(t)+\frac{1}{2} S_{K}^{\alpha}(h)^{2}\left(D_{K}^{\alpha}\right)^{2} z(t)+O\left(S_{K}^{\alpha}(h)^{3}\right) .
$$

The numerical solution of the fractal differential equation is obtained by utilizing Equation (33), which might be called fractal Euler method.

Considering conjugacy of ordinary calculus and fractal calculus, the fractal local truncation error (FLTE) is given by

$$
F L T E=z\left(t_{0}+h\right)-z\left(t_{1}\right)=\left.\frac{1}{2} S_{K}^{\alpha}(h)^{2}\left(D_{K}^{\alpha}\right)^{2} z(t)\right|_{t=t_{0}}+O\left(S_{K}^{\alpha}(h)^{3}\right),
$$

Equation (35) is valid, if we have

$$
\forall t \in K, \exists M>0,\left|\left(D_{K}^{\alpha}\right)^{3} z(t)\right|<M .
$$


Recalling conjugacy of ordinary calculus and fractal calculus, the fractal global truncation error is given by

$$
\forall t \in K, \exists N>0,\left|\left(D_{K}^{\alpha}\right)^{2} z(t)\right|<N,
$$

and $f$ is the fractal Lipschitz continuous, namely

$$
\left|f\left(S_{K}^{\alpha}(t), z_{1}\right)-f\left(S_{K}^{\alpha}(t), z_{2}\right)\right|<Q\left|z_{1}-z_{2}\right| .
$$

In the same manner, the fractal bounded global truncation error (FGTE) is given by

$$
|F G T E| \leq \frac{S_{K}^{\alpha}(h) N}{2 Q}\left(\exp \left(Q\left(S_{K}^{\alpha}(t)-S_{K}^{\alpha}\left(t_{0}\right)\right)-1\right)\right),
$$

since $S_{K}^{\alpha}(h) \leq h^{\alpha}$ then we obtain

$$
|F G T E| \approx h^{\alpha} .
$$

For this reason, the fractal Euler method is also called $\alpha$-order Euler method.

Example 2. Consider the following fractal Cauchy problem

$$
D_{K}^{\alpha} z(t)=z(t), \quad z(0)=1 .
$$

The exact solution Equation (41) is

$$
z(t)=\exp \left(S_{K}^{\alpha}(t)\right)
$$

Applying Equation (34), we proceed with corresponding difference equation as follows

$$
z_{n+1}(t)=z_{n}(t)+S_{K}^{\alpha}(h) z(t),
$$

so that by using the fractal Euler method to approximate solution of Equation (41) we get

$$
z(t+h)=1+S_{K}^{\alpha}(h) z(t)+\frac{1}{2} S_{K}^{\alpha}(h)^{2}\left(D_{K}^{\alpha}\right)^{2} z(t)+O\left(S_{K}^{\alpha}(h)^{3}\right) .
$$

In Figure 2 as well as in the following figures, the smooth colored lines represent the standard results with $\alpha=1$.

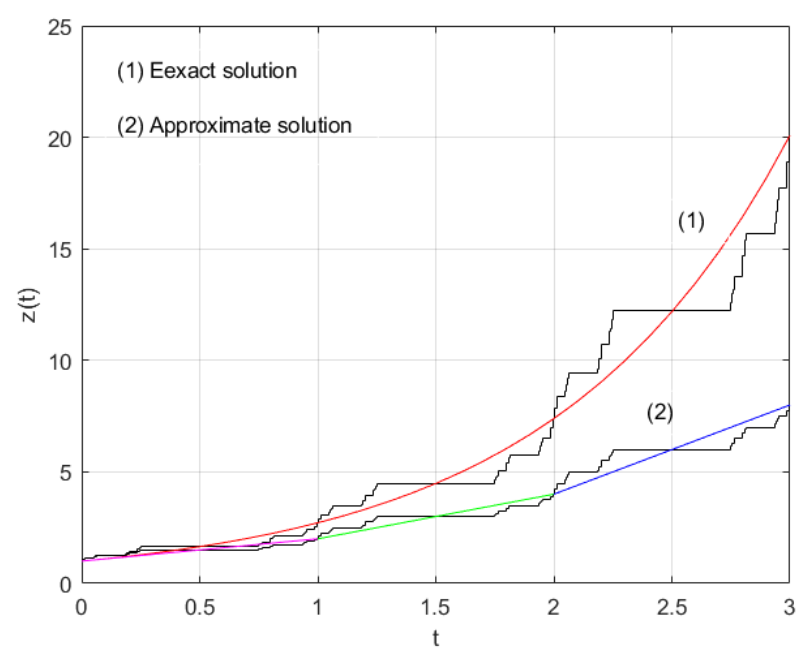

Figure 2. Exact solution and approximate solution using numerical fractal Euler method where step sizes $h=1$ and $\alpha=0.63$. 
Example 3. Consider the fractal time varying harmonic oscillator as follows

$$
\left(D_{K}^{\alpha}\right)^{2} z(t)+z(t)=0, \quad z(0)=1,\left.\quad D_{K}^{\alpha} z(t)\right|_{t=0} .
$$

In view of conjugacy of fractal and standard calculus, the solution of Equation (45) is $z(t)=\cos \left(S_{K}^{\alpha}(t)\right)$. The corresponding fractal difference equation is

$$
\frac{1}{S_{K}^{\alpha}(h)^{2}}\left(z_{n+2}-2 z_{n+1}+z_{n}\right)+z_{n}=0,
$$

with initial conditions

$$
z_{0}=0, z_{1}=0
$$

Using the standard techniques one can get the solution of recurrence relations Equation (46) as follows

$$
z_{n}=\frac{1}{2}\left(\left(1+i S_{K}^{\alpha}(h)\right)^{n}+\left(1-i S_{K}^{\alpha}(h)\right)^{n}\right),
$$

where $z_{n}$ is real number and $i=\sqrt{-1}$. By Equation (28) we have

$$
z_{n}=\cos \left(S_{K}^{\alpha}\left(t_{n}\right)\right)+\frac{1}{2} S_{K}^{\alpha}(h) S_{K}^{\alpha}\left(t_{n}\right) \cos \left(S_{K}^{\alpha}\left(t_{n}\right)\right)+O\left(S_{K}^{\alpha}(h)^{2}\right) .
$$

The approximate solution of Equation (46) for the case $S_{K}^{\alpha}\left(t_{n}\right) S_{K}^{\alpha}(h) \ll 1$ is

$$
z_{n}=\cos \left(S_{K}^{\alpha}\left(t_{n}\right)\right)
$$

and

$$
S_{K}^{\alpha}(h) \rightarrow 0 \Rightarrow z_{n}=\cos \left(S_{K}^{\alpha}\left(t_{n}\right)\right)
$$

It is expected.

\section{Fractal Logistic Equation}

In this section we give the fractal logistic equation and its solution.

Let us consider fractal logistic equation as follows:

$$
D_{K, t}^{\alpha} z(t)=r_{K} z(t)\left(1-\frac{z(t)}{r_{K}^{\prime}}\right)
$$

where $r_{K}$ is called fractal growth parameter and $r_{K}^{\prime}$ is called fractal carrying capacity. Applying conjugacy of fractal calculus and standard calculus we obtain the solution of Equation (52) as follows:

$$
z(t)=\frac{z(0) r_{K}}{z(0)+\left(r_{K}-z(0)\right) \exp \left(-r_{K}^{\prime} S_{K}^{\alpha}(t)\right)},
$$

where $z(0)$ is called initial population. If we consider upper limit of $S_{K}^{\alpha}(t)<t^{\alpha}$ we get

$$
z(t) \simeq \frac{z(0) r_{K}}{z(0)+\left(r_{K}-z(0)\right) \exp \left(-r_{K}^{\prime} t^{\alpha}\right)},
$$

where $z(t)$ is called fractal Logistic function. In Figure 3 we have plotted Equation (53).

The fractal derivative of Equation (53) is

$$
D_{K, t}^{\alpha} z(t)=\frac{z(0) r_{K}\left(r_{K}-z(0)\right) r_{K}^{\prime} \exp \left(-r_{K}^{\prime} S_{K}^{\alpha}(t)\right)}{\left(z(0)+\left(r_{K}-z(0)\right) \exp \left(-r_{K}^{\prime} S_{K}^{\alpha}(t)\right)\right)^{2}},
$$


and its upper bound is

$$
D_{K, t}^{\alpha} z(t) \simeq \frac{z(0) r_{K}\left(r_{K}-z(0)\right) r_{K}^{\prime} \exp \left(-r_{K}^{\prime} t^{\alpha}\right)}{\left(z(0)+\left(r_{K}-z(0)\right) \exp \left(-r_{K}^{\prime} t^{\alpha}\right)\right)^{2}} .
$$

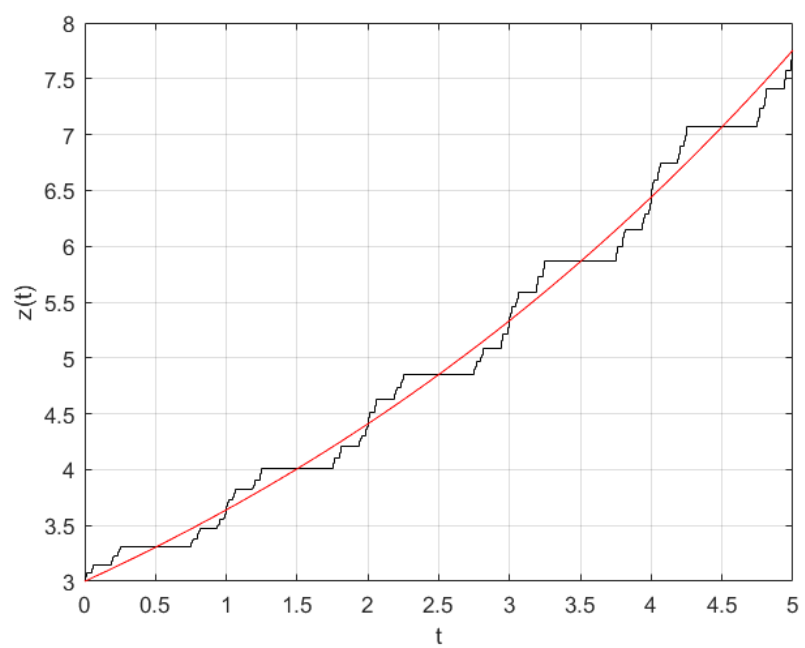

Figure 3. Fractal logistic curve with $\alpha=0.5$ where $\mu=1 / 2 . z(0)=3, r_{K}=100, r_{K}^{\prime}=0.2$.

In Figure 4, we present the upper bound of fractal logistic function and its fractal derivatives by setting different value of $\alpha$.

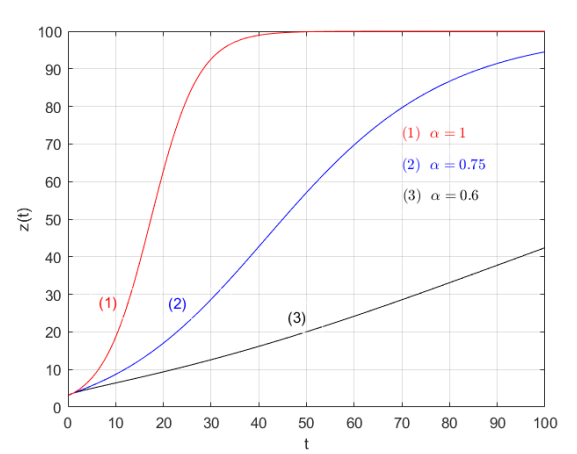

(a) Upper bound function for the value $z(0)=$ 3, $r_{K}=100, r_{K}^{\prime}=0.2$

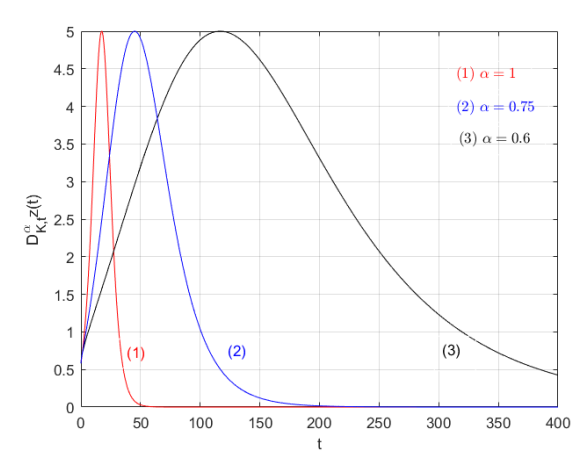

(b) Upper bound of fractal derivative for the values $z(0)=3, r_{K}=100, r_{K}^{\prime}=0.2$

Figure 4. Graph of upper bound of fractal logistic function and its fractal derivative.

The inflection point $\left(t=t_{i p}\right)$ of fractal Logistic function is defined $z\left(t_{i p}\right)=r_{K} / 2$, then we have

$$
S_{K}^{\alpha}\left(t_{i p}\right)=\frac{\ln \left(r_{K} / z(0)-1\right)}{r_{K}^{\prime}} .
$$

Using the upper bound of $S_{K}^{\alpha}(t) \leq t^{\alpha}$, we get

$$
t_{i p}(\alpha)=\left(\frac{\ln \left(r_{K} / z(0)-1\right)}{r_{K}^{\prime}}\right)^{\alpha}
$$

In Figure 5 we have sketched Equation (58) which indicates inflection points versus $\alpha$.

Remark: In figures of this section, we have shown that the power law model for the processes with the fractal structure by using fractal calculus leads to ordinary cases by letting $\alpha=1$. 


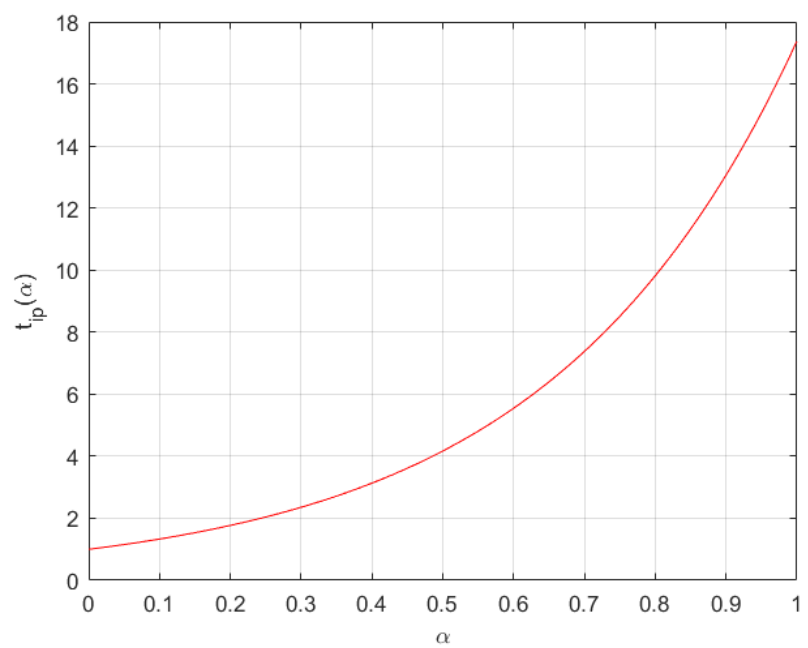

Figure 5. The inflection point as function of $\alpha$ by choosing $z(0)=3, r_{K}=100, r_{K}^{\prime}=0.2$.

\section{Conclusions}

In this paper, we have defined fractal difference equations which are useful for finding numerical methods for solving fractal differential equations. The fractal differential equations and corresponding difference equations were given. Fractal logistic equations and functions were suggested. The upper bound of the fractal logistic function can be considered the most generalized growth model by changing different fractal dimensions. A function of generalized inflection points in terms of fractal dimension was obtained. We have also solved illustrative examples.

Author Contributions: All authors worked jointly and contributed equally.

Funding: This research received no external funding.

Conflicts of Interest: The authors declare no conflict of interest.

\section{References}

1. Mandelbrot, B.B. The Fractal Geometry of Nature; WH Freeman: New York, NY, USA, 1983; Volume 173.

2. Barnsley, M.F. Fractals Everywhere; Academic Press: Cambridge, MA, USA, 2014.

3. Cattani, C.; Pierro, G. On the fractal geometry of DNA by the binary image analysis. Bull. Math. Biol. 2013, 75, 1544-1570. [CrossRef] [PubMed]

4. Heydari, M.H.; Hooshmandasl, M.R.; Ghaini, F.M.; Cattani, C. Wavelets method for solving fractional optimal control problems. Appl. Math. Comput. 2016, 286, 139-154. [CrossRef]

5. Badiali, J.P. Fractal behavior in quantum statistical physics. Phys. Rev. E 1999, 60, 2533. [CrossRef]

6. Demmie, P.N.; Ostoja-Starzewski, M. Waves in fractal media. J. Elast. 2011, 104, 187-204. [CrossRef]

7. Tarasov, V.E. Fractional hydrodynamic equations for fractal media. Ann. Phys. 2005, 318, 286-307. [CrossRef]

8. Lapidus, M.; Lu, H.; van Frankenhuijsen, M. Minkowski dimension and explicit tube formulas for p-adic fractal strings. Fractal Fract. 2018, 2, 26. [CrossRef]

9. Wang, Z.S.; Lu, B.W. The scattering of electromagnetic waves in fractal media. Wave Random Media 1994, 4, 97. [CrossRef]

10. Baish, J.W.; Jain, R.K. Fractals and cancer. Cancer Res. 2000, 60, 3683-3688.

11. Jorgensen, P.E. Analysis and Probability: Wavelets, Signals, Fractals; Springer Science Business Media: Berlin, Germany, 2006; Volume 234.

12. Hinz, M.; Lancia, M.R.; Teplyaev, A.; Vernole, P. Fractal snowflake domain diffusion with boundary and interior drifts. J. Math. Anal. Appl. 2018, 457, 672-693. [CrossRef]

13. Czachor, M. Waves along fractal coastlines: From fractal arithmetic to wave equations. Acta Phys. Pol. 2019, 50, 813-831. [CrossRef] 
14. Riane, N.; David, C. The finite difference method for the heat equation on Sierpinski simplices. Int. J. Comput. Math. 2019, 96, 1477-1501. [CrossRef]

15. Joumaa, H.; Ostoja-Starzewski, M. On the dilatational wave motion in anisotropic fractal solids. Math. Comput. Simul. 2016, 127, 114-130. [CrossRef]

16. Jayasinghe, J.; Andújar, A.; Anguera, J. On the properties of Sierpinski gasket fractal microstrip antennas. Microw. Opt. Technol. Lett. 2019, 61, 772-776. [CrossRef]

17. Djida, J.D.; Nieto, J.J.; Area, I. Nonlocal time porous medium equation with fractional time derivative. Rev. Mat. Complut. 2019, 32, 273-304. [CrossRef]

18. Kigami, J. Analysis on Fractals; Cambridge University Press: Cambridge, UK, 2001.

19. Freiberg, U.; Zahle, M. Harmonic calculus on fractals-a measure geometric approach I. Potential Anal. 2002, 16, 265-277. [CrossRef]

20. Strichartz, R.S. Differential Equations on Fractals: A Tutorial; Princeton University Press: Princeton, NJ, USA, 2006.

21. Falconer, K. Techniques in Fractal Geometry; John Wiley and Sons: Hoboken, NJ, USA, 1997.

22. Barlow, M.T.; Perkins, E.A. Brownian motion on the Sierpinski gasket. Probab. Theory Relat. Fields 1988, 79, 543-623. [CrossRef]

23. Stillinger, F.H. Axiomatic basis for spaces with noninteger dimension. J. Math. Phys. 1977, 18, 1224-1234. [CrossRef]

24. Balankin, A.S. A continuum framework for mechanics of fractal materials I: From fractional space to continuum with fractal metric. Eur. Phys. J. B 2015, 88, 90. [CrossRef]

25. Zubair, M.; Mughal, M.J.; Naqvi, Q.A. Electromagnetic Fields and Waves in Fractional Dimensional Space; Springer: New York, NY, USA, 2012.

26. Liang, W.C.Y. New methodologies in fractional and fractal derivatives modeling. Chaos Soliton Fract. 2017, 102, 72-77.

27. Liang, Y.; Ye, A.Q.; Chen, W.; Gatto, R.G.; Colon-Perez, L.; Mareci, T.H.; Magin, R.L. A fractal derivative model for the characterization of anomalous diffusion in magnetic resonance imaging. Commun. Nonlinear Sci. Numer. Simul. 2016, 39, 529-537. [CrossRef]

28. Nottale, L.; Schneider, J. Fractals and nonstandard analysis. J. Math. Phys. 1998, 25, 1296-1300. [CrossRef]

29. Célérier, M.N.; Nottale, L. Quantum-classical transition in scale relativity. J. Phys. A Math. Gen. 2004, 37, 931-955. [CrossRef]

30. Kolwankar, K.M.; Gangal, A.D. Local fractional Fokker-Planck equation. Phys. Rev. Lett. 1998, 80, 214. [CrossRef]

31. El-Nabulsi, R.A. Classical string field mechanics with non-standard Lagrangians. Math. Sci. 2015, 9, $173-179$. [CrossRef]

32. Yang, H.; Sun, J.; Fu, C. Time-fractional Benjamin-Ono equation for algebraic gravity solitary waves in baroclinic atmosphere and exact multi-soliton solution as well as interaction. Commun. Nonlinear Sci. 2019, 71, 187-201. [CrossRef]

33. Wu, G.C.; Baleanu, D.; Luo, W.H. Lyapunov functions for Riemann-Liouville-like fractional difference equations. Appl. Math. Comput. 2017, 314, 228-236. [CrossRef]

34. Wu, G.C.; Baleanu, D.; Xie, H.P. Riesz Riemann-Liouville difference on discrete domains. Chaos 2016, $26,084308$. [CrossRef]

35. Das, S. Functional Fractional Calculus; Springer Science Business Media: Berlin, Germany, 2011.

36. Verhulst, P.F. Notice sur la loi que la population suit dans son accriossement. Corresp. Math. Phys. 1838, 10, 113-121.

37. Blumberg, A.A. Logistic growth rate functions. J. Theor. Biol. 1968, 21, 42-44. [CrossRef]

38. Cohen, J.E. Population growth and earth's human carrying capacity. Science 1995, 269, 341-346. [CrossRef]

39. Cameron, A.C.; Trivedi, P.K. Regression Analysis of Count Data; Cambridge University Press: Cambridge, UK, 1998.

40. Brenner, J.R. Data analysis made easy with Data Fit. Educ. Chem. Eng. 2006, 40, 60-65.

41. El-Sayed, A.M.A.; El-Mesiry, A.E.M.; El-Saka, H.A.A. On the fractional-order logistic equation. Appl. Math. Lett. 2007, 20, 817-823. [CrossRef]

42. Sweilam, N.H.; Nagy, A.M.; Elfahri, L.E. Nonstandard Finite Difference Scheme For The Fractional Order Salmonella Transmission Model. J. Fract. Calc. Appl. 2019, 10, 197-212. 
43. Area, I.; Losada, J.; Nieto, J.J. A note on the fractional logistic equation. Physica A Stat. Mech. Appl. 2016, 444, 182-187. [CrossRef]

44. Ausloos, M.; Dirickx, M. (Eds.) The Logistic Map and the Route to Chaos: From the Beginnings to Modern Applications; Springer Science Business Media: Berlin, Germany, 2006.

45. Strogatz, S.H. Nonlinear Dynamics and Chaos: With Applications to Physics, Biology, Chemistry, and Engineering; CRC Press: Boca Raton, FL, USA, 2018.

46. Atici, F.M.; Sengul, S. Modeling with fractional difference equations. J. Math. Anal. Appl. 2010, 369, 1-9. [CrossRef]

47. Zhang, B.G.; Zhou, Y. Oscillations of difference equations with several delays. Comput. Math. Appl. 2002, 44, 817-821. [CrossRef]

48. Siegmund, S. Normal forms for nonautonomous difference equations. Comput. Math. Appl. 2003, 45, 1059-1073. [CrossRef]

49. Chen, F.; Luo, X.; Zhou, Y. Existence results for nonlinear fractional difference equation. Adv. Differ. Equ. 2011, 2011, 713201. [CrossRef]

50. Miller, K.S.; Ross, B. Fractional difference calculus, In Proceedings of the International Symposium on Univalent Functions, Fractional Calculus and Their Applications, Koriyama, Japan, 1988; pp. 139-152.

51. Ferreira, R.A.C.; Torres, D.F. Fractional h-difference equations arising from the calculus of variations. Appl. Anal. Discrete Math. 2011, 5, 110-121. [CrossRef]

52. Atici, F.M.; Eloe, P.W. A transform method in discrete fractional calculus. Int. J. Differ. Equ. 2007, 2, $165-176$.

53. Abdeljawad, T.; Al-Mdallal, Q.M.; Jarad, F. Fractional logistic models in the frame of fractional operators generated by conformable derivatives. Chaos Soliton Fract. 2019, 119, 94-101. [CrossRef]

54. Abdeljawad, T.; Al-Mdallal, Q.M. Discrete Mittag-Leffler kernel type fractional difference initial value problems and Gronwall's inequality. J. Comput. Appl. Math. 2018, 339, 218-230. [CrossRef]

55. Singh, J. A new analysis for fractional rumor spreading dynamical model in a social network with Mittag-Leffler law. Chaos 2019, 29, 013137. [CrossRef] [PubMed]

56. Singh, J.; Kumar, D.; Hammouch, Z.; Atangana, A. A fractional epidemiological model for computer viruses pertaining to a new fractional derivative. Appl. Math. Comput. 2018, 316, 504-515. [CrossRef]

57. Parvate, A.; Gangal, A.D. Calculus on fractal subsets of real-line I: Formulation. Fractals 2009, 17, 53-148. [CrossRef]

58. Parvate, A.; Gangal, A.D. Calculus on fractal subsets of real line II: Conjugacy with ordinary calculus. Fractals 2011, 19, 271-290. [CrossRef]

59. Golmankhaneh, A.K.; Baleanu, D. Diffraction from fractal grating Cantor sets. J. Mod. Opt. 2016, 63, $1364-1369$. [CrossRef]

60. Golmankhaneh, A.K.; Fernandez, A.; Golmankhaneh, A.K.; Baleanu, D. Diffusion on middle- $\xi$ Cantor sets. Entropy 2018, 20, 504. [CrossRef]

61. Golmankhaneh, A.K.; Balankin, A.S. Sub-and super-diffusion on Cantor sets: Beyond the paradox. Phys. Lett. A 2018, 382, 960-967. [CrossRef]

62. Golmankhaneh, A.K.; Tunç, C. Sumudu transform in fractal calculus. Appl. Math. Comput. 2019, 350, $386-401$. [CrossRef]

63. Golmankhaneh, A.K.; Fernandez, A. Random Variables and Stable Distributions on Fractal Cantor Sets. Fractal Fract. 2019, 3, 31. [CrossRef]

64. DiMartino, R.; Urbina, W. On Cantor-like sets and Cantor-Lebesgue singular functions. arXiv 2014, arXiv:1403.6554.

(C) 2019 by the authors. Licensee MDPI, Basel, Switzerland. This article is an open access article distributed under the terms and conditions of the Creative Commons Attribution (CC BY) license (http:/ / creativecommons.org/licenses/by/4.0/). 